\title{
Review: brief interventions reduce drinking in patients not seeking treatment
}

\author{
Moyer A, Finney JW, Swearingen CE, et al. Brief interventions for alcohol problems: a meta-analytic review of controlled \\ investigations in treatment-seeking and non-treatment-seeking populations. Addiction 2002 Mar;97:279-92.
} QUESTION: In people with alcohol problems, are brief interventions effective for reducing drinking?

\section{Data sources}

Studies were identified by searching \{Medline, PsycINFO, Dissertation Abstracts, and the Alcohol and Alcohol Problems Science Database (from the National Institute of Alcohol Abuse and Alcoholism) \}* and by scanning citations in relevant studies.

Source of funding: National Institute on Alcohol Abuse and Alcoholism

For correspondence: Dr A Moyer, SUNY Stony Brook, Stony Brook, New York, USA anne.moyer@sunysb.edu.

Abstract and commentary also appear in Evidence-Based Mental Health.

Composite of all drinking-related outcomes for brief interventions for reducing alcohol drinking

\begin{tabular}{lllll} 
Patient group & Comparison & Follow up & $\begin{array}{l}\text { Number } \\
\text { of studies }\end{array}$ & Effect size $(95 \%$ Cl) \\
Not seeking treatment & Brief intervention $v$ control & $\leq 3$ months & 4 & $0.30(0.08$ to 0.52$) \dagger$ \\
\hline & & $>3$ to 6 months & 11 & $0.14(0.08$ to 0.21$) \dagger$ \\
\hline & & $>6$ to 12 months & 23 & $0.24(0.18$ to 0.30$) \dagger$ \\
\hline Seeking treatment & Brief intervention $v$ extended treatment & $\leq 3$ months & 7 & $-0.03(-0.22$ to 0.17$) \ddagger$ \\
\hline & & $>3$ to 6 months & 7 & $0.17(-0.02$ to 0.36$) \ddagger \S$ \\
\hline & & $>6$ to 12 months & 10 & $0.03(-0.10$ to 0.15$) \ddagger \S$ \\
\hline & $>12$ months & 10 & $0.01(-0.12$ to 0.13$) \ddagger \S$ \\
\hline
\end{tabular}

$\dagger$ Effect size favours brief intervention.

‡Not significant.

$\S$ Effect size favours extended treatment. time], abstinence, and drinking without problems). Drinking related outcomes were aggregated, and effect sizes were calculated.

\section{Main results}

56 studies were included. 34 studies compared brief interventions with control conditions in people who were not seeking treatment; $79 \%$ of these studies excluded people with alcohol dependence, heavy drinking, or previous treatment for alcohol problems. A benefit in the aggregate outcome was seen for brief interventions at $\leq 3$ months (4 studies), $>3$ to 6 months ( 11 studies), and $>6$ to 12 months (23 studies), but the effect was not statistically significant at $>12$ months (5 studies) (table). 20 studies compared brief interventions with extended treatments in people who were seeking treatment, and $50 \%$ of these studies excluded people with alcohol dependence, heavy drinking, or previous treatment for alcohol problems. No difference in the aggregate outcome was seen between brief interventions and extended treatment at any time point (table).

\section{Conclusions}

In people with alcohol problems who are not seeking treatment, brief interventions are better than no intervention for reducing drinking. In people seeking treatment for alcohol problems, brief interventions do not differ from extended treatment for reducing drinking.

*Information provided by author.

\section{COMMENTARY}

Brief interventions have consistently been found effective for alcohol-use problems and at-risk drinking behaviours. ${ }^{12}$ However, application of these findings to primary care has been hampered by an absence of adequate cost-effectiveness data. The 2 studies by Moyer and Fleming $e t$ al add to our understanding of the treatment effects and costs

In their meta-analysis, Moyer et al take a conceptual step forward by grouping the brief intervention studies according to patient type: those who were opportunistically identified as having problem drinking behaviours and those who sought alcohol treatment. This distinction turned out to yield a clear difference in the size of the outcome effects seen in these 2 populations. The carefully done analyses and tables provide elegant and clear data on effect sizes and data homogeneity.

In the analysis by Moyer $e t$ al of the studies concerning patients not seeking treatment, a small to moderate treatment effect was observed after $\leq 4$ provider contacts; this effect was equivalent to a $10 \%$ to $20 \%$ increase in the number of patients achieving a favourable response. This effect seems worthy at first glance, but is it significant enough to warrant widespread implementation of the screening and intervention and commitment of new resources to augment existing health promotion activities? To address these concerns, relevant cost-effectiveness data are needed from multiple studies. Given the large number of competing care demands on primary care providers, we must prove that the rather modest $10 \%$ to $20 \%$ improvement rate translates into meaningful, clinical benefits.

To this end, the study by Fleming et al provides some encouraging evidence that the cost-benefit ratio may justify the investment. The effectiveness of delivering the intervention in routine, daily practice seemed to save $\$ 4.30$ for every $\$ 1$ spent; however, the $95 \%$ CI of the cost-benefit ratio was wide, ranging from 0.6 to 8.0 , and the $\mathrm{p}$ value was only 0.08 . This favourable trend needs to be replicated in other large scale studies to be convincing.

The cost of screening in the study by Fleming et al represented $50 \%$ of the expense. If the prevalence of problem drinking is lower in a different study population, the percentage of resources going towards screening and away from intervention will be increased. The incidence of at-risk drinkers was $14 \%$ in the study by Fleming $e t$ al but only $8 \%$ in a similar study by Senft $e t a l^{3}$; consequently, the cost-benefit ratio should be lower and the project less attractive. In reality, the study by Senft $e t$ al failed to find any savings or outcome differences at 12 months. 


\title{
A brief intervention reduced alcohol drinking for up to 48 months in problem drinkers
}

\author{
Fleming MF, Mundt MP,French MT, et al. Brief physician advice for problem drinkers: long-term efficacy and benefit-cost \\ analysis. Alcohol Clin Exp Res 2002 Jan;26:36-43.
}

\author{
QUESTION: In people with problem drinking, is a brief intervention effective for \\ reducing drinking in the long term?
}

\section{Design}

Randomised (unclear allocation concealment*), unblinded,* controlled trial with 48 months of follow up (Project TrEAT [Trial for Early Alcohol Treatment]).

\section{Setting}

64 primary care physician offices from 17 clinics in 10 southern Wisconsin counties, USA.

\section{Patients}

774 patients who were 18 to 65 years of age $(62 \%$ men) and drank a large amount of alcohol weekly $(>14$ drinks [ $168 \mathrm{~g}$ of alcohol] for men, $>11$ drinks [132 $\mathrm{g}$ of alcohol] for women) or drank $>5$ drinks on $\geq 4$ occasions in the previous 30 days. Patients were excluded if they had formal alcohol treatment in the previous year or a history of alcohol withdrawal, were pregnant, or had suicide ideation. Follow up was $83 \%$ at 48 months.

\section{Intervention}

Patients were allocated to a brief intervention $(n=392)$ or no intervention $(\mathrm{n}=382)$. All patients received a general health booklet containing prevention messages. Patients in the intervention group received two 15 minute sessions 1 month apart with their physician and two 5 minute follow up telephone calls from office nurses. The protocol was scripted and included a workbook with tasks for patients to complete at home.

\section{Main outcome measures}

Alcohol use, healthcare use, motor vehicle and legal events, mortality, and costs (1993 US dollars).

\section{Main results}

Analysis was by intention to treat. The intervention group had lower rates of 7 day alcohol use $(p=0.002)$ and a lower mean number of binge drinking episodes in the previous 30 days $(\mathrm{p}<0.001)$ than did the control group. Heavy drinking ( $>20$ drinks/wk for men or $>13$ drinks/wk for women) was reduced more in the treatment group than in the control group in the first 2 years $(\mathrm{p}<0.001)$, but the reduction was similar by 48 months, from $47 \%$ to $22 \%$ in the intervention group and from $49 \%$ to $26 \%$ in the control group. Binge drinking ( $>5$ drinks on 1 occasion) in the previous 30 days was reduced from $85 \%$ to $64 \%$ in the intervention group and from $87 \%$ to $70 \%$ in the control group ( $\mathrm{p}<0.001$ for overall 48 mo treatment effect). The intervention group had fewer days in hospital $(420 v 664 \mathrm{~d}, \mathrm{p}<0.05)$ and fewer arrests for controlled substance or liquor violations $(2 v 11, \mathrm{p}<0.05)$; differences in healthcare use or motor vehicle or other legal events were not statistically significant. The groups did not differ for mortality at 48 months. When costs were analysed from a medical perspective, the net benefit was not statistically significant ( $\$ 546,95 \%$ CI $-\$ 71$ to $\$ 1164)$; from a societal perspective, the net benefit was statistically significant $(\$ 7780$, CI $\$ 894$ to $\$ 14668)$.

\section{Conclusions}

In patients with problem drinking, a brief intervention reduced alcohol drinking for up to 48 months. A net benefit was seen when costs were analysed from a societal perspective.

*See glossary.
Sources of funding: Robert Wood Johnson Foundation and National Institutes of Health

For correspondence:

Dr M F Fleming,

University of

Wisconsin-Madison, Madison, Wisconsin USA.

mfleming@fammed.wisc.edu.

Abstract and commentary also appear in Evidence-Based Mental Health.

\section{COMMENTARY continued from previous page}

Brief interventions seem to be inexpensive and require little staff input. However, when applied broadly, they require daily, systematic staff effort, including the screening of many normal patients to identify each at-risk person ( $7: 1$ ratio in the study by Fleming $e t$ al). For a health maintenance organisation with 300000 adult members, such as Kaiser Permanente of Colorado, an anticipated 42000 members would qualify as problem drinkers, if the prevalence is $14 \%$. Kaiser's 250 primary care physicians would need to devote two 15 minute visits per patient to replicate Fleming's intervention. If all members were to be screened within 4 years, it would take $1 \%$ of every physician's time to do the intervention, cost roughly $\$ 10 \mathrm{~m}$ ( $\$ 166 /$ patient, adjusted to 2002 dollars), and save perhaps $\$ 42 \mathrm{~m}$. Before implementing a project affecting so many physicians and members, complementary studies that replicate the positive findings by Fleming $\mathrm{et}$ al are needed.

Finally, on a serendipitous note, the data suggest a unique opportunity to increase the cost-benefit ratio by including the savings from motor vehicle crash expenses in the calculations. An additional $\$ 7171$ was probably saved for each $\$ 166$ spent on brief interventions (95\% CI $\$ 396$ to $\$ 13965$ ). These potential savings may be of interest to auto insurers. Perhaps a unique collaboration could be created between auto and health insurance companies to finance brief intervention programmes for the mutual benefit of their subscribers. Is this a place where the rubber meets the road?

Allan Graham, MD

Kaiser Permanente Health Plan of Colorado, Denver, Colorado, USA

1 Bien TH, Miller WR, Tonigan JS. Brief intervention for alcohol problems: a review. Addiction 1993;88:315-36. Senft RA, Polen MR, Freeborn DK, et al. Brief intervention in a primary care setting for hazardous drinkers. Am J Prev Med 1997;13:464-70. 